\title{
Photoinduced Vibrations Drive Ultrafast Structural Distortion in Lead Halide Perovskite
}

\author{
Hong-Guang Duan, ${ }^{\perp}$ Vandana Tiwari, ${ }^{\perp}$ Ajay Jha, ${ }^{\perp}$ Golibjon R. Berdiyorov, Alexey Akimov,
} Oriol Vendrell, Pabitra K. Nayak, Henry J. Snaith, Michael Thorwart, Zheng Li,* Mohamed E. Madjet,* and R. J. Dwayne Miller*

Cite This: J. Am. Chem. Soc. 2020, 142, 16569-16578

Read Online

ACCESS | 네 Metrics \& More | 回 Article Recommendations | sl Supporting Information

ABSTRACT: The success of organic-inorganic perovskites in optoelectronics is dictated by the complex interplay between various underlying microscopic phenomena. The structural dynamics of organic cations and the inorganic sublattice after photoexcitation are hypothesized to have a direct effect on the material properties, thereby affecting the overall device performance. Here, we use ultrafast heterodyne-detected two-dimensional (2D) electronic spectroscopy to reveal impulsively excited vibrational modes of methylammonium (MA) lead iodide perovskite, which drive the structural distortion after photoexcitation. Vibrational analysis of the measured data allows us to

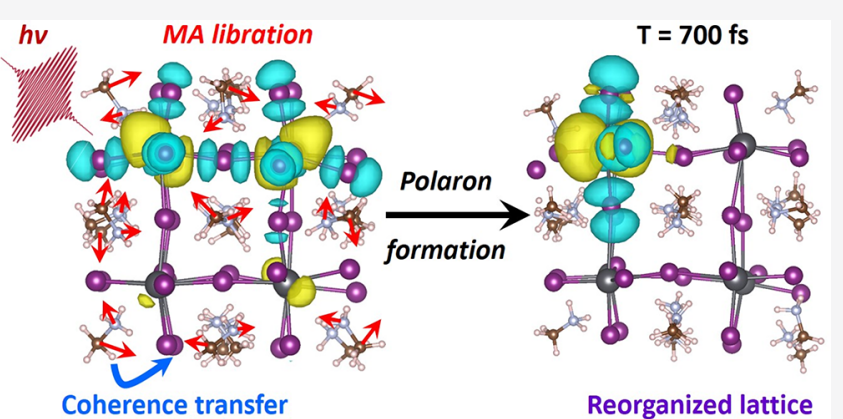
monitor the time-evolved librational motion of the MA cation along with the vibrational coherences of the inorganic sublattice. Wavelet analysis of the observed vibrational coherences reveals the coherent generation of the librational motion of the MA cation within $\sim 300 \mathrm{fs}$ complemented with the coherent evolution of the inorganic skeletal motion. To rationalize this observation, we employed the configuration interaction singles (CIS), which support our experimental observations of the coherent generation of librational motions in the MA cation and highlight the importance of the anharmonic interaction between the MA cation and the inorganic sublattice. Moreover, our advanced theoretical calculations predict the transfer of the photoinduced vibrational coherence from the MA cation to the inorganic sublattice, leading to reorganization of the lattice to form a polaronic state with a long lifetime. Our study uncovers the interplay of the organic cation and inorganic sublattice during formation of the polaron, which may lead to novel design principles for the next generation of perovskite solar cell materials.

\section{INTRODUCTION}

On the quest for a low-cost and easily processable photoactive material, organic-inorganic lead halide perovskites (LHPs) have emerged as a class of material with tremendous potential. Photovoltaics based on LHPs have displayed a remarkable increase in power conversion efficiencies (PCE) as compared to other technologies in the past decade advancing to $24.2 \%$ based on a solid polycrystalline perovskite. ${ }^{1-6}$ Largely due to their easy solution processability, large carrier diffusion length, and high photoluminescence quantum yield, ${ }^{7-9}$ perovskites have also found their application in other areas such as photodetectors and lasing. ${ }^{3,10-14}$ Despite the success of the material to reach macroscopic performance scales such as the highly efficient PCE, a comprehensive understanding of the underlying microscopic phenomena of charge generation following photoexcitation is still far from being understood.

To unravel the microscopic mechanisms of the success of perovskite in photoinduced charge generation, numerous experimental and theoretical works have been reported focusing on understanding the elementary photophysical processes in LHPs. ${ }^{15-23}$ One of the important unresolved questions pertaining to perovskite photophysics is the origin of long charge carrier lifetimes using processing methods that generally lead to midgap states. Those act as efficient recombination centers, although the charge carrier mobilities in LHPs have been reported to be modest, i.e., only 50-100 $\mathrm{cm}^{2} \mathrm{~V}^{-1} \mathrm{~s}^{-1}$. $8,24,25$ This puzzling observation has attracted enormous attention by various research groups. To understand the underlying reasons for the long charge carrier lifetime in $\mathrm{CH}_{3} \mathrm{NH}_{3} \mathrm{PbI}_{3}$, $\mathrm{Zhu}$ and co-workers used a combination of the time-resolved optical Kerr effect and photoluminescence spectroscopy to compare the ultrafast hot-electron cooling dynamics in perovskites with different organic cations. ${ }^{26}$ There

Received: April 11, 2020

Published: September 1, 2020 
(a)

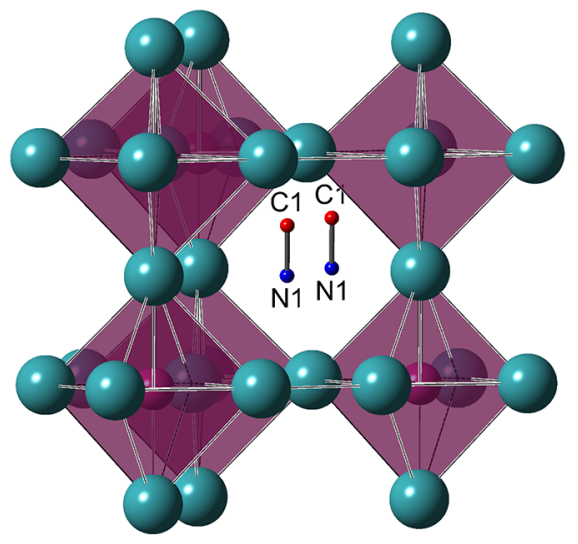

(b)

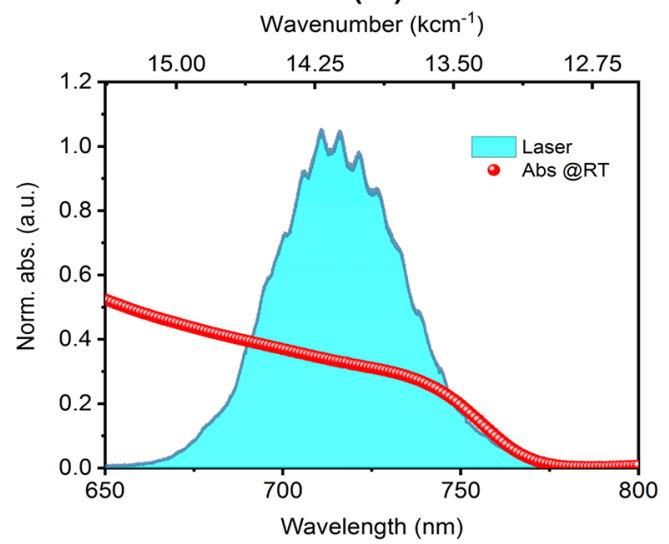

(c)

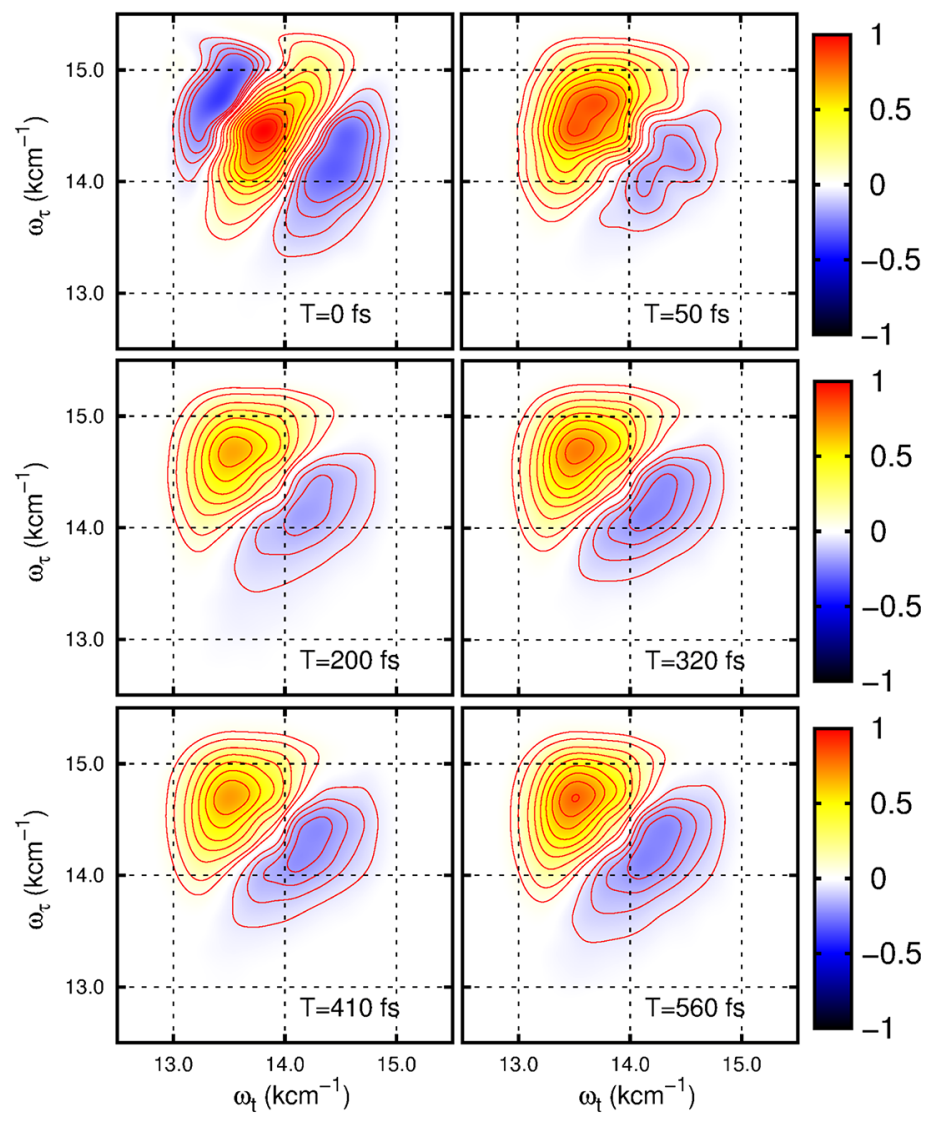

Figure 1. (a) Ground-state structure of tetragonal $\mathrm{MAPbI}_{3}$. (b) Steady-state absorption spectrum (red circle) of perovskite at room temperature and laser spectrum (light blue area). (c) Time evolution of 2D electronic spectra (real part) at selected waiting times. Magnitude of the spectra decays with growing waiting time. Interestingly, in 2D spectra, the oscillation of the amplitude from 200 to 560 fs can be clearly observed. Positive and negative amplitudes indicate the ground-state bleach and excited-state absorption, respectively.

the reorientational motion of the MA cation has been identified to form the charge-screening effect which protects the energetic carriers via formation of a large polaron on time scales competitive with that of ultrafast thermalization and recombination. In addition, based on the resonant and offresonant excitations, the existence of a large polaron formation has been further supported in order to explain the modest charge mobilities of electrons in perovskite. ${ }^{17,27}$ However, their latest observations confirmed the Kerr effect, and the coherent oscillations can be well explained by a complex artifact arising from the static birefringence of the material. Combination of the experiments and calculations has proposed that the deformation of the $\mathrm{PbBr}_{3}{ }^{-}$sublattice is mainly responsible for the polaron formation. The intrinsic effects of organic cations on the band-edge charge carrier lifetime were studied by time-resolved photoluminescence of LHPs as a function of temperature, ${ }^{28}$ which also supports the hypothesis of formation of a large polaron. To capture the lattice displacement induced by photocarrier generation in LHPs, recently LHPs have also been studied using impulsive vibrational spectroscopy ${ }^{29}$ and electron diffraction. ${ }^{30}$ Due to the proposed role of the organic cation in perovskite photophysics, its vibrational dynamics has been extensively studied by various spectroscopic approaches. $^{36-39}$ Raman and photoluminescence spectroscopy have been used to investigate the structure-function relationships in $\mathrm{MAPbI}_{3}$. The librational motion of the MA cation is found to be strongly coupled to the $\mathrm{PbI}_{6}$ perovskite octahedra by a hydrogen bond. ${ }^{39}$ Moreover, the vibrational dynamics of the inorganic sublattice have been studied during the process of polaron formation. ${ }^{40}$ The observed low-frequency modes of the $\mathrm{Pb}-\mathrm{I}$ bending and stretching vibrations result in the charge separation in the LHPs. Interestingly, resonant $\mathrm{THz}$ phonon excitation shows direct evidence of the mode-driving band gap in the LHPs, ${ }^{41}$ which demonstrates the correlation of the band gap and the $\mathrm{Pb}-\mathrm{I}-\mathrm{Pb}$ angle bending vibrations. Advanced theoretical calculations have uncovered that polaron formation is induced by the structural disorder resulting mainly from thermal distortions of the inorganic sublattice. They reduce the overlap between the electron and the hole wave functions, and the probability of bimolecular recombination is then lowered by 2 orders of magnitude. ${ }^{31}$ In addition to polaron formation, Rashba spin-orbit interaction has been considered to enhance the carrier lifetime in LHPs. ${ }^{32}$ On the basis of first-principles calculations and a Rashba spin-orbital model, Zheng and coworkers suggested that the recombination rate is reduced due to spin-forbidden transitions. The subsequent transient absorption measurement is consistent with the existence of a giant Rashba splitting in 2D LHPs. ${ }^{33}$ This Rashba effect has also been reported in LHPs thin films. ${ }^{34}$ In addition, the vibrational coherence of the librational motion of the MA cation has been calculated in a multiunit-cell model of perovskite, $^{35}$ showing that a ferroelectric domain wall is constructed to reduce the electron-hole recombination. Despite these enormous efforts, there has been no conclusive 
(a)

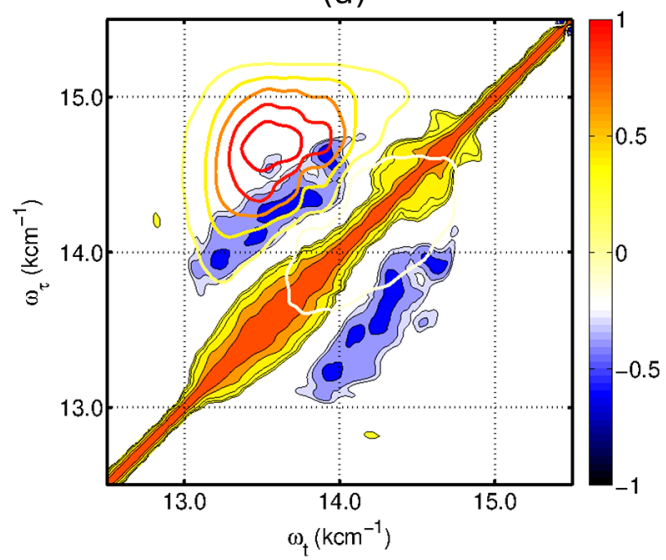

(c)

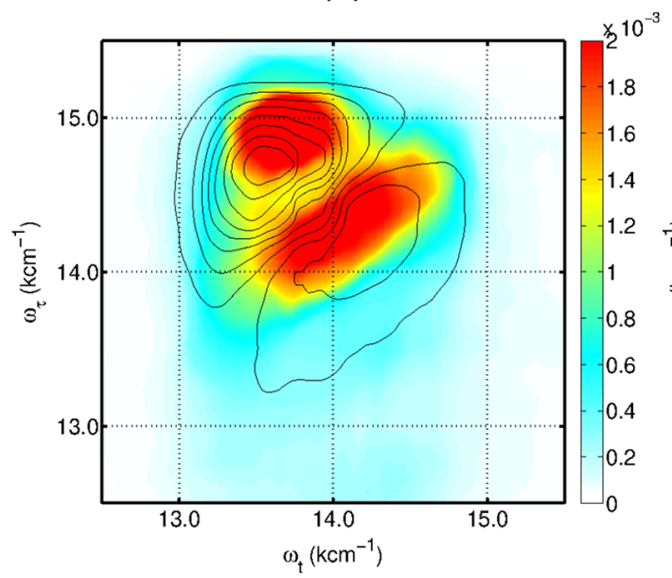

(b)

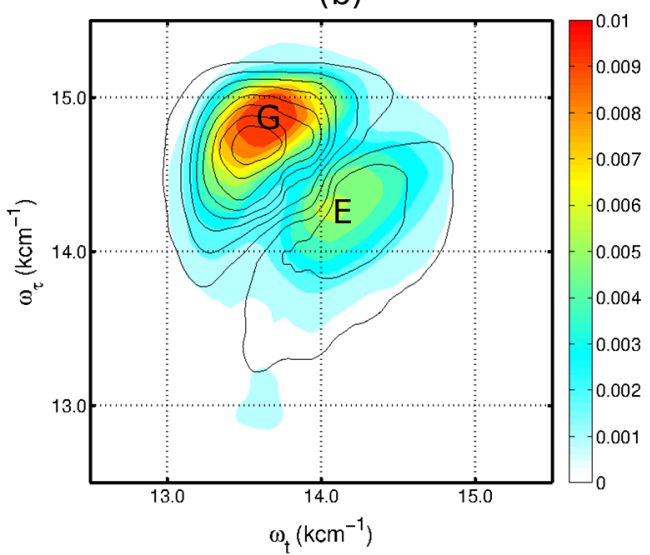

(d)

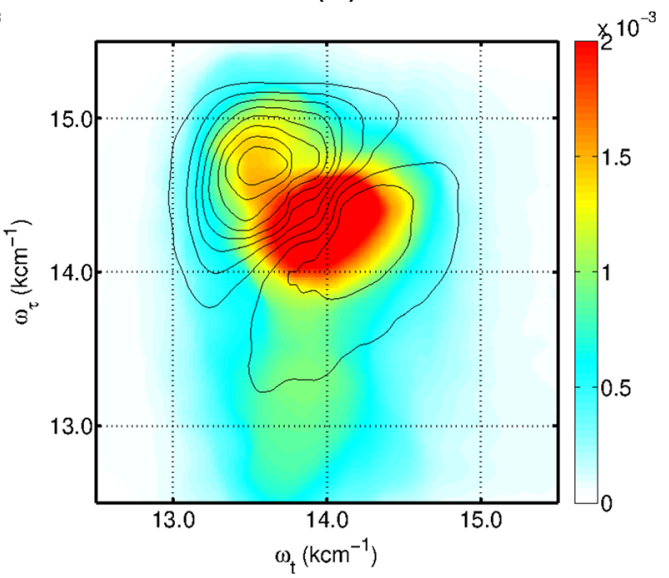

Figure 2. (a) Two-dimensional correlation spectrum after analyzing correlated cross peaks along the diagonal. Two-dimensional electronic spectrum at $T=200 \mathrm{fs}$ is shown as contours for comparison. (b, c, and d) Two-dimensional power spectra at frequencies of 157,65 , and $81 \mathrm{~cm}^{-1}$, respectively. For comparison, $2 \mathrm{D}$ spectrum at $T=200 \mathrm{fs}$ is plotted as a black contour. For data analysis, we extract the kinetics of the selected peaks at $\mathrm{G}$ and $\mathrm{E}$ in $\mathrm{b}$ to examine their coherent dynamics in GSB and ESA, respectively.

experimental report capturing the dynamics of the organic cation and its interaction with the inorganic sublattice during formation of the large polaron. Such an analysis can reveal the degree to which the cation is involved and whether this potential microscopic mechanism for the long carrier lifetime is sufficiently strong.

To resolve the ultrafast structural dynamics of the polaron formation in $\mathrm{CH}_{3} \mathrm{NH}_{3} \mathrm{PbI}_{3}$, we studied the coherent vibrational dynamics of the inorganic sublattice and its correlation with the librational motion of the organic cation using ultrafast twodimensional electronic photoecho spectroscopy (2DES). 2DES measures the third-order nonlinear response originating from the interaction of the sample with three resonant electric fields. The response is commonly plotted as a function of both the excitation and the probe frequencies. In contrast to conventional pump-probe schemes, 2DES spectrally decomposes the optical signal into the excitation and detection windows. In this way $2 \mathrm{DES}$ is able to disentangle the underlying transitions to provide the distinct selectivity of transient optical signatures and the transfer pathways. Due to these advantages, 2DES has recently been used to study the exciton dynamics and the carrier thermalization processes in LHPs. ${ }^{19,42,43}$ Here, we judiciously tuned our laser spectrum to capture the motion of the nuclear wave packet in the electronically excited state by the spectrally disentangled excited-state absorption peak. Subsequent data analysis shows clear evidence of the coherent generation of the librational motion of the MA cation and the skeletal motion of the inorganic sublattice. The subsequent wavelet analysis enables us to directly monitor the formation and the coherence of the librational motion, resolved on an ultrafast time scale of $300 \mathrm{fs}$. In addition, it also reveals that the coherent generation of the librational motion of the MA cation is complemented by the motion of the inorganic sublattice in perovskite. Furthermore, we report advanced theoretical calculations to capture the coherent evolution of the librational modes of the MA cation. Our theory reveals a strong anharmonic interaction between the MA cation and the skeletal motion of the inorganic sublattice in pervoskite. This plays a significant role to drive the excited-state wave packet to form a polaron state. On the basis of this combination of experimental and theoretical efforts, we conclude that the interaction of the librational motion of the organic cation with the inorganic sublattice is responsible for the formation of the polaronic state.

\section{RESULTS}

The solution-processed $\mathrm{CH}_{3} \mathrm{NH}_{3} \mathrm{PbI}_{3}$ is prepared (details are described in the Methods) and grown on a quartz substrate with $1 \mathrm{~mm}$ thickness. For the optical measurement, the sample film is mounted in the cryostat (Oxford Instrument) with vacuum conditions to avoid degradation by moisture. Figure 1a shows the molecular structure of tetragonal $\mathrm{CH}_{3} \mathrm{NH}_{3} \mathrm{PbI}_{3}$ with 


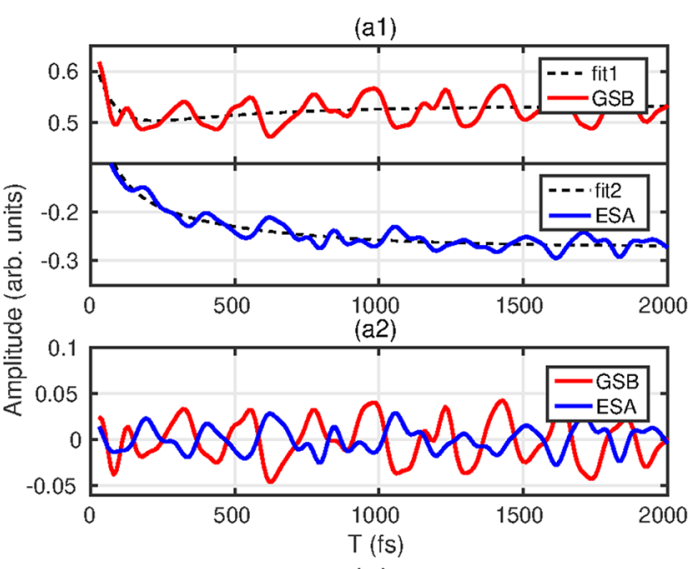

(c)

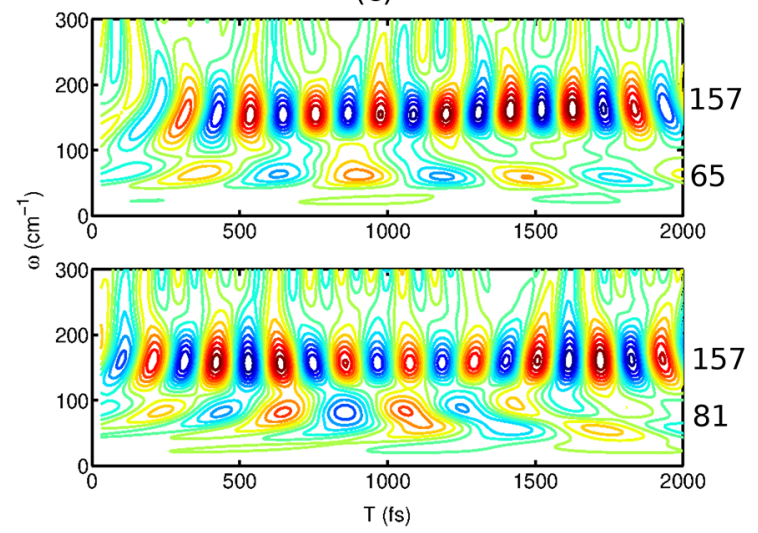

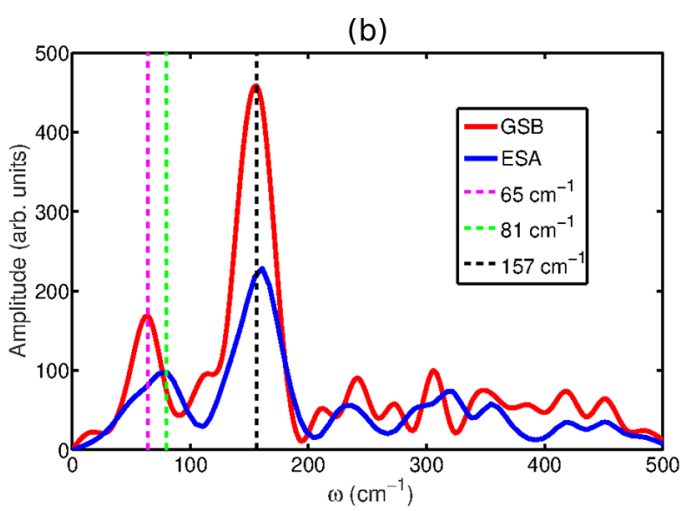

(d)
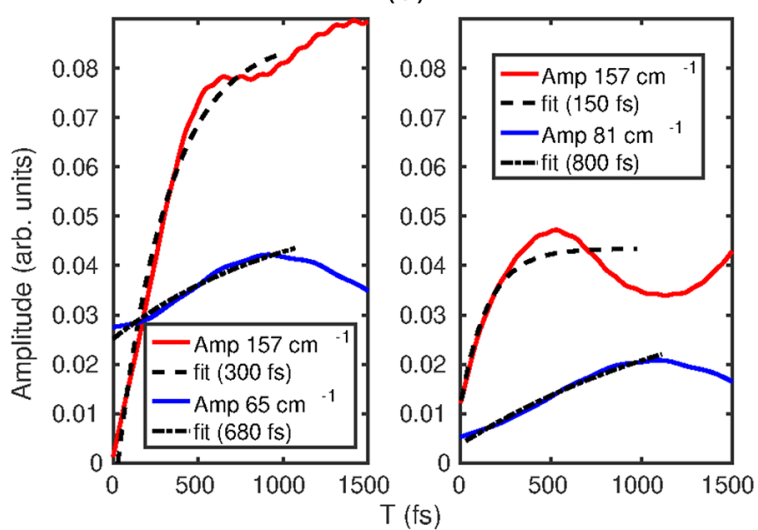

Figure 3. (a1) Traces at GSB (red solid line) and ESA (blue solid line). Their coordinates are marked as G and E separately in Figure $2 \mathrm{~b}$. These traces are fitted by the global fitting approach in a1, and residuals are obtained after removing kinetics. (a2) Polished residuals are shown as red (G) and blue (E) solid lines after removing the high-frequency noise. (b) Identified vibrational frequencies in the power spectrum. Red (G) and blue (E) solid lines show the vibrational modes at 65,81 , and $157 \mathrm{~cm}^{-1}$. (c) Wavelet analysis of residuals on GSB and ESA. (d) Coherent dynamics of the 65, 81, and $157 \mathrm{~cm}^{-1}$ modes from GSB (upper panel) and ESA (lower panel) are presented at the left and right part. In GSB (left), coherent generation of 65 and $157 \mathrm{~cm}^{-1}$ shows an increase in amplitude on the time scale of 680 and $300 \mathrm{fs}$, respectively. Coherent dynamics of modes at 81 and $157 \mathrm{~cm}^{-1}$ show the amplitude increase on time scales of 800 and $150 \mathrm{fs}$ in the ESA contribution (right), respectively.

the MA cation. In addition, the steady-state absorption spectrum of $\mathrm{CH}_{3} \mathrm{NH}_{3} \mathrm{PbI}_{3}$ is shown in Figure $1 \mathrm{~b}$. The laser spectrum used in the present measurements is marked by the light-blue shaded area.

Two-Dimensional Electronic Photon Echo Spectroscopy. To study the exciton and free carrier dynamics after photoexcitation, we measure the $2 \mathrm{D}$ electronic spectra of $\mathrm{CH}_{3} \mathrm{NH}_{3} \mathrm{PbI}_{3}$ at room temperature $(296 \mathrm{~K})$. The details of our home-built $2 \mathrm{D}$ spectrometer and the acquisition procedures are described in the Methods. The real part of the $2 \mathrm{D}$ spectra at selected waiting times is shown in Figure 1c. At $T=0 \mathrm{fs}$, the peak in the $2 \mathrm{D}$ spectrum shows clear evidence of elongation along its diagonal, which indicates the presence of strong inhomogeneous broadening. Moreover, the strong absorption features at zero waiting time ( $T=0 \mathrm{fs}$ ) overlap with the central (positive) peak, which induces a dramatic shift of the central peak to the upper left. At $T=50 \mathrm{fs}$, the magnitude of the $2 \mathrm{D}$ spectrum is strongly reduced and the peaks show less elongation along the diagonal, which indicates a rapid reduction of the strong inhomogeneous broadening within this initial time range. The inhomogeneity of the peaks is hard observe at $T=200 \mathrm{fs}$, and the amplitude of the peaks is further reduced compared that at $T=50 \mathrm{fs}$. The initial dynamics of the photoexcited perovskite within 200-300 fs have been attributed to lattice reorganization and carrier thermalization processes (electron cooling dynamics). ${ }^{42,43}$ The shape of the peaks does not significantly change in the $2 \mathrm{D}$ spectra with increasing waiting time after 200 fs. Interestingly, the magnitude of the peaks in the $2 \mathrm{D}$ spectra at $320 \mathrm{fs}$ is larger than that at $200 \mathrm{fs}$, and the same behavior is observed from 410 to $560 \mathrm{fs}$, which clearly illustrates the presence of oscillations with large amplitude in the $2 \mathrm{D}$ electronic spectra.

Coherent Vibrational Dynamics. To examine the oscillations observed in 2DES, we first collect a threedimensional (3D) set of data consisting of $2 \mathrm{D}$ spectra with varying waiting times $T$. To retrieve the oscillatory signal of the 2D spectra, we perform a global fitting by exponential functions on the $3 \mathrm{D}$ data and analyze the oscillations along the excitation and detection frequencies of the residuals obtained after removing the global kinetics. To assign the origin of the observed oscillations, we perform a $2 \mathrm{D}$ correlation analysis of the $2 \mathrm{D}$ residual maps in which the oscillations of the correlated two peaks are analyzed along the diagonal direction in the $2 \mathrm{DES}$. The resulting $2 \mathrm{D}$ correlation map is shown in Figure $2 \mathrm{a}$, and the details of this analysis are given in the SI. We observe negative peaks with a strong amplitude which are present along the diagonal in the $2 \mathrm{D}$ correlation spectrum. These negative peaks provide strong evidence of anticorrelated oscillations at the presented coordinates in the 2DES. It has been demonstrated that anticorrelated oscillations in $2 \mathrm{D}$ spectra originate from underlying vibrational coherences. ${ }^{44,45}$ 
Thus, this correlation analysis uncovers the vibrational origin of the coherence in the observed oscillations in the 2DES data.

Next, we analyzed the frequencies of these vibrational oscillations by employing a Fourier transform of the 2D residuals obtained after removing the kinetics by the global fitting approach. We identified a few key modes with the largest amplitudes and plotted the absolute values of the observed distinct frequencies along $\omega_{\tau}$ and $\omega_{t}$ in $2 \mathrm{D}$ power spectra. We show selected $2 \mathrm{D}$ power spectra for the frequencies of 157,65 , and $81 \mathrm{~cm}^{-1}$ in Figure $2 \mathrm{~b}, 2 \mathrm{c}$, and $2 \mathrm{~d}$. In addition, two retrieved $2 \mathrm{D}$ power spectra with modes at 33 and $48 \mathrm{~cm}^{-1}$ are shown in the SI. The black contour of the $2 \mathrm{D}$ spectrum at $200 \mathrm{fs}$ is overlaid with the $2 \mathrm{D}$ power maps in order to identify the location of the oscillations. This comparison reveals that the amplitude of the 2D power map at $157 \mathrm{~cm}^{-1}$ overlays with the ground-state bleach (GSB) and the excited-state absorption (ESA) features in the 2D electronic spectrum. The vibrational mode of $157 \mathrm{~cm}^{-1}$ shows the strongest magnitude in the $2 \mathrm{D}$ power spectra with the time period of $208 \mathrm{fs}$, which perfectly agrees with the amplitude fluctuation observed in Figure 1c. The strong amplitude in Figure $2 \mathrm{~b}$ shows clear evidence of strong vibronic coupling of this key mode to the electronic transitions in our 2DES. Moreover, in this $2 \mathrm{D}$ power spectrum, we observe two separated peaks, marked by $\mathrm{G}$ and $\mathrm{E}$, which are connected by a node. It implies that the dynamical oscillations at these two peaks have anticorrelated phases, which agrees with the observations of the $2 \mathrm{D}$ correlation analysis in Figure 2a. In Figure $2 \mathrm{c}$ and $2 \mathrm{~d}$, we clearly observe that the magnitudes of vibrations are mainly distributed in the GSB and ESA regions with the separation of a node. To further explore the origin of this coherence, we extract the kinetic traces at the maximum amplitude of the $G$ and $E$ peaks in Figure $2 b$ and plot them as red $(G)$ and blue (E) solid lines in Figure 3al. To retrieve residuals we use the global fitting approach to fit their kinetics and show the result of the fit as black dashed lines. The residuals of the raw data are shown as red and blue dashed lines in the lower part of Figure S6 in the SI. Moreover, the high-frequency noise is removed by a tukey-window Fourier transform, and the polished residuals are presented as red and blue solid lines for GSB and ESA in Figure 3a2, respectively. We observe that the phases of the oscillations shown by the red and blue lines are anticorrelated, which demonstrates the validity of our analysis reported in the $2 \mathrm{D}$ correlation spectrum in Figure 2a. The details of the tukey-window Fourier transform are described in the SI.

To distinguish the vibrations of the electronic ground or excited states, we construct a theoretical model with three electronic states and identify the optical signals of the GSB and ESA by assigning the optical transitions from the ground to the first excited state and the transition from the first to the second excited state, respectively. The $2 \mathrm{D}$ electronic spectra are calculated for different waiting times, and the retrieved vibrational coherences from the GSB and ESA peaks are further analyzed to extract the phase relation. More details on the calculations of the $2 \mathrm{D}$ spectra are described in the SI. On the basis of our theoretical calculations, the anticorrelated oscillations from the GSB and ESA demonstrate the validity of our model and show the separation of vibrations from the electronic ground and excited states (see Figure S3 in the SI). By this we are able to monitor the electronic and vibrational dynamics from the ground and excited states by tracking the kinetics of the GSB and ESA peaks. To retrieve their oscillation frequencies, we perform the Fourier transform of the two residuals and plot the power spectra in Figure $3 \mathrm{~b}$. We clearly identify three key modes at frequencies of 65,81 , and 157 $\mathrm{cm}^{-1}$, which are marked by the magenta, green, and black dashed lines, respectively. Due to the lifetime broadening of modes at frequencies of 65 and $81 \mathrm{~cm}^{-1}$, the low-frequency modes at 33 and $48 \mathrm{~cm}^{-1}$ are not well resolved in Figure $3 \mathrm{~b}$. It shows a strong mode at $157 \mathrm{~cm}^{-1}$ both in the GSB and in the ESA. However, in the region of low frequency $\left(<100 \mathrm{~cm}^{-1}\right)$, slightly different frequencies in the GSB and ESA are found. To obtain the lifetime of this coherent generation, we analyze the data from the GSB and ESA regions using the wavelet analysis. The spectra obtained are shown in Figure $3 \mathrm{c}$ (details of the analysis are described in the SI). We extract the kinetics of the oscillations at 65 and $157 \mathrm{~cm}^{-1}$ and obtain the time evolution of the amplitude by fitting the sine function with exponential decay. The time-evolved amplitudes are shown in the left part of Figure 3d. The amplitudes for the cases at 65 and $157 \mathrm{~cm}^{-1}$ are extracted from GSB and plotted as blue and red solid lines, respectively. The exponential fit of the data reveals a coherent generation of vibrations at $157 \mathrm{~cm}^{-1}$ within $300 \mathrm{fs}$. Using a similar analysis, we observe the enhancement of the vibrational coherence of $65 \mathrm{~cm}^{-1}$ with a time scale of 680 fs. In addition, we perform the same data analysis for the traces of 81 and $157 \mathrm{~cm}^{-1}$ in the ESA part and plot the results in the right panel of Figure $3 \mathrm{~d}$. We find a coherent generation of vibrations at 81 and $157 \mathrm{~cm}^{-1}$ within 800 and $150 \mathrm{fs}$, respectively.

Computational Analysis. To identify the origin of the experimentally observed vibrational coherences and to simulate their dynamics, we performed theoretical calculations of the photoexcitation process in $\mathrm{CH}_{3} \mathrm{NH}_{3} \mathrm{PbI}_{3}$ (details of the computations are provided in the Methods). First, we perform DFT calculations in the tetragonal phase of perovskite. Figure 4a shows the difference of the electron localization functions for the ground and excited states. The excited state has been simulated by adding $10^{19} \mathrm{~cm}^{-3}$ excess electrons, which is close to the charge density under experimental conditions. Detailed calculations of carrier densities with varying excitation energies were discussed in our pervious work. ${ }^{19}$ As we show in Figure $4 \mathrm{a}$, the electrons are mainly localized on the $\mathrm{Pb}$ atom and this excess charge strongly interacts with the positively charged $\mathrm{NH}_{3}{ }^{+}$group. It forces the dipole of the MA cation to rotate toward the $\mathrm{Pb}$ atom (this is supported by the argument elaborated in the Discussion). To capture the subsequent structural dynamics, we carry out the excited-state moleculardynamics calculation for a model system with the $\mathrm{MA}_{28} \mathrm{~Pb}_{12} \mathrm{I}_{52}$ cluster for the time evolution of 2 ps. We show the dominant principal axis as the representative motion of the model system in Figure $4 \mathrm{~b}-\mathrm{e}$, in which librational motions of the MA cation at $158 \mathrm{~cm}^{-1}$ and the $\mathrm{Pb}-\mathrm{I}$ lattice distortion appear as the major components of the dynamical processes. Specified configurations of the skeletal vibrations are shown in Figure $4 \mathrm{c}$ and $4 \mathrm{~d}$ with frequencies of 61 and $87 \mathrm{~cm}^{-1}$, respectively. In addition, our simulations show two low-frequency modes at 37 and $47 \mathrm{~cm}^{-1}$; they are described in the SI. Moreover, our dynamical calculations reveal the coherent generation of the librational motion of the MA cation at $158 \mathrm{~cm}^{-1}$. In Figure $4 \mathrm{e}$, we observe that the magnitude of the vibration at $158 \mathrm{~cm}^{-1}$ (marked by the black dashed line) gradually increases with evolving time and reaches its maximum at $300 \mathrm{fs}$. This time scale of the coherent generation perfectly agrees with the time scale revealed by the experimental observations reported 
(a) ELF

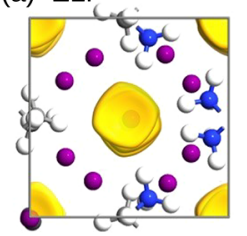

(c)

$61 \mathrm{~cm}^{-1}(\mathrm{I}-\mathrm{Pb}-\mathrm{I}$ bend)

(e)
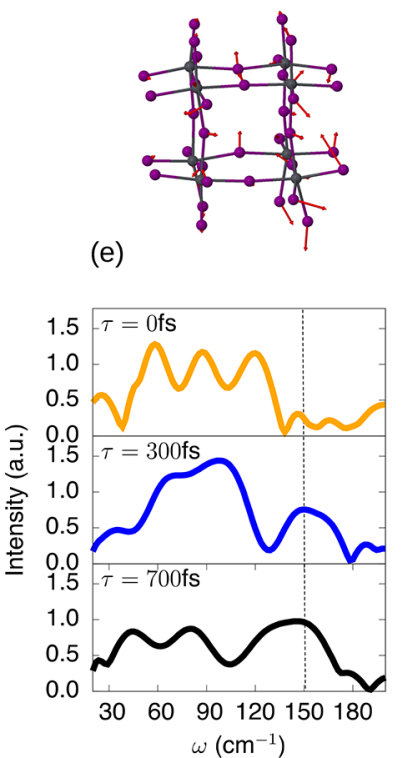

(b) $158 \mathrm{~cm}^{-1}$ MA libration

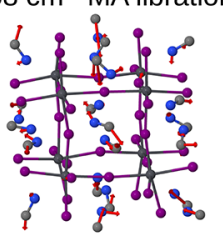

(d)

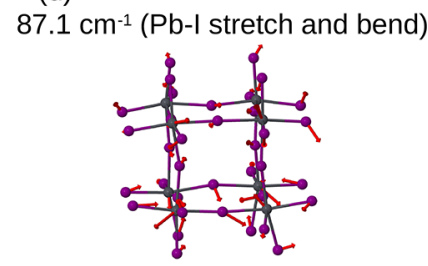

(f)

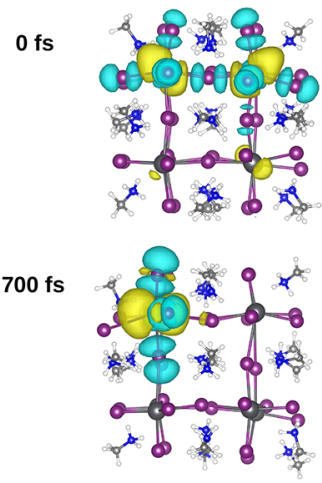

Figure 4. (a) Difference of the electron localization function with $10^{19}$ $\mathrm{cm}^{-3}$ excess electrons compared to its ground state. Magnitude of its isosurface is 0.03. (b) Molecular configuration of perovskite with the librational motions of the MA cation at $158 \mathrm{~cm}^{-1}$. (c and d) Vibrational coherence of the inorganic sublattice is present in the lowfrequency region, 61 and $87 \mathrm{~cm}^{-1}$, respectively. (e) Time evolution of the vibrational coherence after photoexcitation. Librational motion of the MA cation $\left(158 \mathrm{~cm}^{-1}\right)$ is generated on a time scale of $300 \mathrm{fs}$. Moreover, the low-frequency $\left(<100 \mathrm{~cm}^{-1}\right)$ shift is presented within $\sim 700$ fs after photoexcitation. (f) On the basis of the ground-state equilibrium structure, the initial electron density is strongly delocalized after photoexcitation. Electron density is localized after reaching equilibrium at $700 \mathrm{fs}$. MA cation rotates its molecular axis to align to the localized charge on the $\mathrm{Pb}$ atom. In addition, the sublattice is slightly distorted compared to the equilibrium configuration of the ground state.

above. In addition, our theoretical calculations uncover an anharmonic interaction between the strong coherent oscillations of the MA cation and the skeletal motion of the inorganic sublattice in perovskite. Induced by this interaction, the vibrational coherences of the skeletal motion are strongly modulated during the coherent generation of the librational motion of the MA cation, which is clearly shown in Figure 4e. These structure-based calculations allow us to identify the atomic motions of the MA cation, which strongly interact with the adjacent skeletal $(\mathrm{I}-\mathrm{Pb}-\mathrm{I})$ motions after being subject to the optical transition from the valence to the conduction band. Thus, along with the librational motion of the MA cation, the calculated vibrational motions corresponding to the inorganic sublattice observed at $37,47,61$, and $87 \mathrm{~cm}^{-1}$ actively participate in the process of polaron formation.

Discussion. On the basis of the experimental and computational approaches described above, we conclude that the electron-sublattice interaction in the photoexcited

tetragonal $\mathrm{CH}_{3} \mathrm{NH}_{3} \mathrm{PbI}_{3}$ leads to formation of a large polaron and involves the interplay of different vibrational frequencies representing organic and inorganic sublattices' motion. Specifically, the intense vibrational coherences of the methyl librational mode at $158 \mathrm{~cm}^{-1}$ dominate the initial dynamics at early times of 200-300 fs. In order to clarify the underlying mechanism for the buildup of the librational amplitude of the MA cation, we analyzed the temporal evolution of the difference of the electronic density between the excited and the ground states, $\Delta \rho(\mathrm{t})=\rho_{\mathrm{ES}}(\mathrm{t})-\rho_{\mathrm{GS}}(\mathrm{t})$, for the $\mathrm{MA}_{28} \mathrm{~Pb}_{12} \mathrm{I}_{52}$ model system subject to the same excited-state structures in the MD trajectory. As shown in Figure $4 \mathrm{f}$, both the initially created electron and the hole are delocalized subject to the perovskite structure close to the tetragonal symmetric equilibrium ground state. The localization of the excess electron on the $\mathrm{Pb}$ atom aligns the $\mathrm{MA}$ cation by rotating its molecular axis. This generates the strong signal of the vibrational coherence of the MA cation on an ultrafast time scale of $300 \mathrm{fs}$. The strong interaction transfers the coherence from the librational motion of the organic cation to the motion of the sublattice of the $\mathrm{I}-\mathrm{Pb}-\mathrm{I}$ skeleton. On the basis of our simulations, we find that this transfer of coherence is finished within a time span of $1 \mathrm{ps}$, which can be observed in Figure $4 \mathrm{f}$ at $700 \mathrm{fs}$. We show the detailed time evolution of electronic charge on the skeleton of perovskite in the SI (Figure S7). The localization of the charge density, in turn, enhances the MA librational amplitude. With this redistributed charge, the skeletal motion of $\mathrm{CH}_{3} \mathrm{NH}_{3} \mathrm{PbI}_{3}$ slightly changes its frequencies to reach the (metastable) local potential minimum of the stabilized polaron.

It is important to mention that recent studies on the excited state of LHP focus only on the skeletal modes that are comprised of the motion of the inorganic sublattice, ${ }^{29,40}$ but the information on the dynamics of the organic cation has not been given its due importance. Nevertheless, it could be that in the ground state the organic cation is electronically decoupled from the inorganic sublattice, as shown by a study using an infrared-pump electronic-probe measurement. ${ }^{46}$ However, after photoexcitation, the reorganization of the lattice due to polaron formation leads to a significant interaction between the organic cation and the $\mathrm{PbI}_{3}{ }^{-}$sublattice. In fact, this interaction provides the necessary stabilization for the polaronic state that effectively localizes the carrier by reducing the electron-hole carrier overlap which blocks carrier recombination. The resulting electrostatic screening, unique to the strong ionic character of LHPs, greatly distinguishes this class of materials from other semiconductors. It is this ultrafast intense polaron formation that is responsible for the observed long carrier lifetime in these materials. This holds even for simple methods of sample preparation that are normally associated with high defect densities and rapid recombination losses. This class of materials is distinct from other polar and ionic semiconductors in that the structure comprised of an inorganic and a labile organic ionic sublattice leads to a localized charge density, an exceptionally strong coupling to the lattice, and the noted ultrafast large polaron formation. Moreover, this polaron formation has been even reported without the role of organic cation in $\mathrm{CsPbBr}_{3} ;{ }^{47}$ however, the underlying mechanism is different from this study, and it should be further explored in the subsequent studies. 


\section{CONCLUSIONS}

We studied the coherent dynamics of photoexcitations in tetragonal $\mathrm{CH}_{3} \mathrm{NH}_{3} \mathrm{PbI}_{3}$ at room temperature. On the basis of the high sensitivity of heterodyne-detected 2D electronic spectroscopy, we provide evidence of quantum coherences, which emerge after photoexcitation of an electron from the valence band to the hot free-carrier band. A 2D correlation analysis reveals the origin of the vibrational coherences in 2DES. On the basis of the phase analysis on the GSB and ESA peaks, the dominating modes with frequencies of 65,81 , and $157 \mathrm{~cm}^{-1}$, which are coupled to the electronic states, are identified. Moreover, the strong magnitude of the oscillations manifests a strong vibronic coupling of these key modes to the electronic transitions during photoexcitation. On the basis of a wavelet analysis, we further reveal that the key mode of 157 $\mathrm{cm}^{-1}$ is gradually generated on an ultrafast time scale of $300 \mathrm{fs}$. The amplitude of the low-frequency modes at 65 and $81 \mathrm{~cm}^{-1}$ are enhanced during the process of the coherent generation of the $157 \mathrm{~cm}^{-1}$ mode. Supported by our advanced theoretical calculations, we are able to assign the key mode of $157 \mathrm{~cm}^{-1}$ to the librational motion of the MA cation and our theoretical prediction of the coherent generation and increasing amplitude of the cation motion occurring within the time scale of $300 \mathrm{fs}$. This perfectly agrees with our experimental observation. Moreover, we identified the modes at 33, 48, 65, and 81 $\mathrm{cm}^{-1}$ as the key modes related to vibrational coherence of the skeletal motion of $\mathrm{PbI}_{3}^{-}$. A structure-based approach allows us to capture evidence of a strong interaction between the MA cation and the inorganic sublattice. This interaction induces a coherent transfer of excitation from the librational motion of the MA cation to the skeletal motion of $\mathrm{PbI}_{3}{ }^{-}$in which the sublattice motion of $\mathrm{PbI}_{3}^{-}$strongly modulates the photogenerated wave packet dynamics on the surface of the electronic excited states. Moreover, we theoretically captured the wave packet dynamics which assists polaron formation by localizing the electron density on the skeletal structure of the $\mathrm{Pb}$ atom. Thus, our study provides a new comprehensive understanding of the wave packet motion, which induces the vibrationally coherent polaron formation process, and hence unravels the underlying mechanism of the charge generation and protection in $\mathrm{CH}_{3} \mathrm{NH}_{3} \mathrm{PbI}_{3}$. On the basis of this finding, one may anticipate rational design principles for future improvement of perovskite materials.

\section{METHODS}

Experimental Setup. Ultrashort coherent pulses were generated by a home-built nonlinear optical parametric amplifier pumped by a commercial femtosecond laser Pharos (Light Conversion). A broadband spectrum with a line width of $100 \mathrm{~nm}$ (fwhm) was centered at $13800 \mathrm{~cm}^{-1}$ such that an overlap with the near-infrared region of the absorption spectrum of perovskites is achieved. The excitation pulse was further compressed to the Fourier transform limit with a duration of $16 \mathrm{fs}$ by the combination of a prism pair (F2) and a deformable mirror (OKO Technologies). Their temporal profiles were characterized by means of a frequency-resolved optical grating (FROG). The measured FROG traces were analyzed using a commercial program FROG3 (Femtosecond Technologies). The 2D spectra were collected in an all-reflective $2 \mathrm{D}$ spectrometer based on a diffractive optic (Holoeye) with a phase stability of $\lambda / 160$ whose configuration is described elsewhere. ${ }^{58}$ Further components were the Sciencetech spectrometer model 9055 and a highly sensitive CCD linear array camera (Entwicklungsbüro Stresing). The 2D spectra were collected at each fixed waiting time $T$ by scanning the delay time $\tau=t_{1}-t_{2}$ in the range of $[-128 \mathrm{fs}, 128 \mathrm{fs}]$ with a delay step of $1 \mathrm{fs}$. At each delay point, 200 spectra were averaged to reduce the noise ratio. The waiting time $T=t_{3}-t_{2}$ was linearly scanned in the range of $0-2 \mathrm{ps}$ in steps of $10 \mathrm{fs}$. The energy of the excitation pulse is limited to $5 \mathrm{~nJ}$ with $1 \mathrm{kHz}$ repetition rates for room-temperature measurements. To check the pump dependence, we also carried out measurements with different pump energies ( 5 and $10 \mathrm{~nJ}$ ). We observed that the basic features of the $2 \mathrm{D}$ electronic spectra do not change at different energy fluence. However, the cooling dynamics of hot electrons are more significant at the higher energy flux. Three pulses are focused on the sample with the spot size $100 \mu \mathrm{m}$, and the photon echo signal was generated at the phase-matching direction. To avoid oxidation, the sample was kept in the cryostat under vacuum conditions $\left(1.7 \times 10^{-7}\right.$ $\mathrm{mPa}$ ) at room temperature. To verify the reproducibility of the results, measurements were performed on different spots on the perovskite films as well as with films prepared from three different batches. All showed similar spectral features.

Sample Preparation and Measuring Condition. Acetonitrile (ACN), gamma butyrolactone (GBL), methylammonium solution, chloroform, and $\mathrm{PbI}_{2}$ were procured from Sigma-Aldrich and used as received. MAI was procured from Dyesol. Tetragonal single crystals of MAPbI3 were prepared by a method described in ref 59 and used as a precursor material. The precursor solution was prepared by dissolving single crystals of MAPbI3 in an acetonitrile and methyl amine solution. ${ }^{60}$ The final concentration of the solution was $0.5 \mathrm{M}$. The perovskite films were prepared by spin coating the precursor solution at $2000 \mathrm{rpm}$ on quartz substrates for $45 \mathrm{~s}$ in a $\mathrm{N}_{2}$-purged drybox. The UV-vis spectrum of the thin film was taken on a Carry 300 UV-vis spectrometer.

Theoretical Model. The density functional theory calculations with periodic boundary conditions were performed using QuantumATK. To mimic the charge localization on the $\mathrm{Pb}$ atom, electrons with a density of $10^{19} \mathrm{~cm}^{-3}$ have been injected into the system. By this we can study the motion of electrons in the electronically excited state of perovskite. ${ }^{48}$ Characterization of the localized charge in Figure 4a is done by the electron localization function, ${ }^{49}$ which quantifies the probability of finding a second like-spin electron in the vicinity of an electron at a given space point.

The Born-Oppenheimer excited-state molecular dynamics was computed for a model system with $\mathrm{MA}_{28} \mathrm{~Pb}_{12} \mathrm{I}_{52}$ cluster for $2 \mathrm{ps}$ on the configuration interaction singles (CIS) level with the SBKJC effective core potential ${ }^{50}$ using the Chemical Dynamics Toolkit $(\mathrm{CDTK})^{55}$ and GAMESS. ${ }^{56}$ The MD trajectories were initiated from the structures in the ground-state tetragonal $(14 / \mathrm{mcm})$ geometry after equilibration at room temperature. Its lowest unoccupied orbital is highly localized on a specific $\mathrm{Pb}$ atom, which agrees with the results from PBC-DFT. We analyzed the excited-state $\mathrm{MD}$ trajectories by their principal component, ${ }^{57}$ which was obtained by diagonalizing the covariance matrix $C=\left\langle\mathbf{x x}^{T}\right\rangle$. Here, $\mathbf{x}=\mathbf{r}-\langle\mathbf{r}\rangle$ are the atomic displacement vectors in the $3 \mathrm{~N}$ dimensional configuration space for structures in the MD trajectories. To obtain the dynamics of the dominant principal motions, we project the vibrations onto the principal axes to show the substantial structural transition from the initial geometry to the excited-state polaronic geometry within a time of 1 ps. Because the skeletal modes of the $\mathrm{Pb}-\mathrm{I}$ lattice $\left(<100 \mathrm{~cm}^{-1}\right)$ are similar in the ground and excited states, ${ }^{40}$ we decompose this principal axis vector of the atomic displacements with vibrational eigenvectors of the ground-state perovskite by taking their inner products. We obtain the vibrational spectrum from the time evolution of the atomic velocities $\vec{v}_{k}(t)$ in the excited state with consideration of the large amplitude motion $^{51-53}$

$$
\begin{aligned}
I(\tau, \omega) & =\mathcal{F}_{\mathrm{t}}[I(\tau, t)](\omega) \\
& =\mathcal{F}_{\mathrm{t}}\left\{\frac{1}{N_{\mathrm{at}}} \sum_{k}^{N_{\mathrm{at}}}\left\langle\mathbf{v}_{k}(\tau) \cdot \mathbf{v}_{k}(\tau+t) \exp \left(-\alpha t^{2}\right)\right\rangle\right\}(\omega)
\end{aligned}
$$

where $\mathcal{F}_{\mathrm{t}}$ denotes the Fourier transform, $N_{\mathrm{at}}$ is the number of atoms, and the parameter $\alpha$ is chosen to suppress the autocorrelation function to effectively select the vibrational modes that are present 
around $\tau$. In this way, the temporal evolution of vibrational modes can be revealed by both their frequencies and amplitudes. ${ }^{52-54}$

\section{ASSOCIATED CONTENT}

\section{(s) Supporting Information}

The Supporting Information is available free of charge at https://pubs.acs.org/doi/10.1021/jacs.0c03970.

Global fitting approach, correlation analysis in 2D electronic spectra, 2D power spectra of vibrational modes at 33 and $48 \mathrm{~cm}^{-1}$, vibrational coherences in $2 \mathrm{D}$ electronic spectroscopy, Tukey-window Fourier transform, raw traces of the GSB and ESA peaks, wavelet analysis, time evolution of electronic charge, and charge localization at different carrier densities (PDF)

\section{AUTHOR INFORMATION}

\section{Corresponding Authors}

Zheng Li - State Key Laboratory for Mesoscopic Physics, School of Physics, Peking University, Beijing 100871, China; Max Planck Institute for the Structure and Dynamics of Matter, Hamburg 22761, Germany; (i) orcid.org/0000-0002-54839149; Email: zheng.li@pku.edu.cn

Mohamed E. Madjet - Qatar Environment and Energy Research Institute, Qatar Foundation, Hamad Bin Khalifa University, Doha, Qatar; Email: mmadjet@hbku.edu.qa

R. J. Dwayne Miller - Max Planck Institute for the Structure and Dynamics of Matter, Hamburg 22761, Germany; The Hamburg Center for Ultrafast Imaging, Hamburg 22761, Germany; The Departments of Chemistry and Physics, University of Toronto, Toronto, Ontario M5S 3H6, Canada; ○ orcid.org/0000-0003-0884-0541; Email: dmiller@ lphys2.chem.utoronto.ca

\section{Authors}

Hong-Guang Duan - Max Planck Institute for the Structure and Dynamics of Matter, Hamburg 22761, Germany; I. Institut für Theoretische Physik, Universität Hamburg, Hamburg 20355, Germany; The Hamburg Center for Ultrafast Imaging, Hamburg 22761, Germany; ○ orcid.org/0000-0001-65890890

Vandana Tiwari - Max Planck Institute for the Structure and Dynamics of Matter, Hamburg 22761, Germany; Department of Chemistry, University of Hamburg, Hamburg 20146, Germany

Ajay Jha - Max Planck Institute for the Structure and Dynamics of Matter, Hamburg 22761, Germany

Golibjon R. Berdiyorov - Qatar Environment and Energy Research Institute, Qatar Foundation, Hamad Bin Khalifa University, Doha, Qatar

Alexey Akimov - Department of Chemistry, State University of New York at Buffalo, Buffalo, New York 14260, United States

Oriol Vendrell - Physikalisch-Chemisches Institut, Universität Heidelberg, Heidelberg 69120, Germany

Pabitra K. Nayak - Department of Physics, University of Oxford, Clarendon Laboratory, Oxford OX1 3PU, United Kingdom; TIFR Centre for Interdisciplinary Sciences, Tata Institute of Fundamental Research, Hyderabad 500046, India

Henry J. Snaith - Department of Physics, University of Oxford, Clarendon Laboratory, Oxford OX1 3PU, United Kingdom; (1) orcid.org/0000-0001-8511-790X

Michael Thorwart - I. Institut für Theoretische Physik, Universität Hamburg, Hamburg 20355, Germany; The
Hamburg Center for Ultrafast Imaging, Hamburg 22761, Germany; () orcid.org/0000-0002-5837-0835

Complete contact information is available at: https://pubs.acs.org/10.1021/jacs.0c03970

\section{Author Contributions}

${ }^{\perp}$ H.-G.D., V.T., and A.J.: These authors contributed equally to this work.

\section{Notes}

The authors declare the following competing financial interest(s): H.J.S. is the co-founder and CSO of Oxford PV Ltd., a company that is commercializing perovskite photovoltaic technologies.

\section{ACKNOWLEDGMENTS}

This work was supported by the Max Planck Society and the Excellence Cluster "The Hamburg Center for Ultrafast Imaging-Advanced Imaging of Matter" of the Deutsche Forschungsgemeinschaft. The authors thank V. I. Prokhorenko for help with the $2 \mathrm{D}$ setup and providing $2 \mathrm{D}$ data analysis software and Marcelo Carignano for helpful discussions on the excited-state MD simulation. P.K.N. and H.J.S.T. acknowledge support from the UK Engineering and Physical Sciences Research Council (grant no EP/P032591/1). M.E.M. and G.B. acknowledge the Research Computing Center at Texas A\&M University in Qatar and a grant from the Computational Materials and Processes Center of the Qatar Environment Energy Research Institute (CMP-QEERI) under Project ID P19003.

\section{REFERENCES}

(1) Nayak, P. K.; Mahesh, S.; Snaith, H. J.; Cahen, D. Photovoltaic Solar Cell Technologies: Analysing the State of the Art. Nat. Rev. Mater. 2019, 4, 269-285.

(2) Green, M. A.; Dunlop, E. D.; Levi, D. H.; Hohl-Ebinger, J.; Yoshita, M.; Ho-Baillie, A. W. Y. Solar Cell Efficiency Tables (version 54). Prog. Photovoltaics 2019, 27, 565-575.

(3) Yang, W. S.; Noh, J. H.; Jeon, N. J.; Kim, Y. C.; Ryu, S.; Seo, J.; Seok, S. I. High-performance Photovoltaic Perovskite Layers Fabricated Through Intramolecular Exchange. Science 2015, 348, 1234-1237.

(4) Jeon, N. J.; Noh, J. H.; Yang, W. S.; Kim, Y. C.; Ryu, S.; Seo, J.; Seok, S., II Compositional Engineering of Perovskite Materials for High-performance Solar Cells. Nature 2015, 517, 476-480.

(5) For record cell efficiencies, see: Best Research-Cell Efficiencies; https://www.nrel.gov/pv/cell-efficiency.html.

(6) Ball, J. M.; Lee, M. M.; Hey, A.; Snaith, H. J. Low-temperature Processed Meso-superstructured to Thin-film Perovskite Solar Cells. Energy Environ. Sci. 2013, 6, 1739-1743.

(7) Burschka, J.; Pellet, N.; Moon, S. J.; Humphry-Baker, R.; Gao, P.; Nazeeruddin, M. K.; Grätzel, M. Sequential Deposition as a Route to High-performance Perovskite-sensitized Solar Cells. Nature 2013, 499, 316-319.

(8) Stranks, S. D.; Eperon, G. E.; Grancini, G.; Menelaou, C.; Alcocer, M. J. P.; Leijtens, T.; Herz, L. M.; Petrozza, A.; Snaith, H. J. Electron-hole Diffusion Lengths Exceeding 1 Micrometer in an Organometal Trihalide Perovskite Absorber. Science 2013, 342, 341344

(9) Xing, G.; Mathews, N.; Sun, S.; Lim, S. S.; Lam, Y. M.; Grätzel, M.; Mhaisalkar, S.; Sum, T. C. Long-range Balanced Electron- and Hole-transport Lengths in Organic-inorganic $\mathrm{CH}_{3} \mathrm{NH}_{3} \mathrm{PbI}_{3}$. Science 2013, 342, 344-347.

(10) Zhu, H.; Fu, Y.; Meng, F.; Wu, X.; Gong, Z.; Ding, Q.; Gustafsson, M. V.; Trinh, M. T.; Jin, S.; Zhu, X. Y. Lead Halide 
Perovskite Nanowire Lasers with Low Lasing Thresholds and High Quality Factors. Nat. Mater. 2015, 14, 636-642.

(11) Lee, M. M.; Teuscher, J.; Miyasaka, T.; Murakami, T. N.; Snaith, H. J. Efficient Hybrid Solar Cells Based on Meso-superstructured Organometal Halide Perovskites. Science 2012, 338, 643647.

(12) Kim, H.-S.; Lee, C.-R.; Im, J.-H.; Lee, K.-B.; Moehl, T.; Marchioro, A.; Moon, S.-J.; Humphry-Baker, R.; Yum, J.-H.; Moser, J. E.; Gratzel, M.; Park, N.-G. Lead Iodide Perovskite Sensitized Allsolid-state Submicron Thin Film Mesoscopic Solar Cell with Efficiency Exceeding 9\%. Sci. Rep. 2012, 2, 591.

(13) Etgar, L.; Gao, P.; Xue, Z.; Peng, Q.; Chandiran, A. K.; Liu, B.; Nazeeruddin, M. K.; Grätzel, M. Mesoscopic $\mathrm{CH}_{3} \mathrm{NH}_{3} \mathrm{PbI}_{3} / \mathrm{TiO}_{2}$ Heterojunction Solar Cells. J. Am. Chem. Soc. 2012, 134, 1739617399.

(14) Lin, Q.; Armin, A.; Burn, P. L.; Meredith, P. Filterless Narrowband Visible Photodetectors. Nat. Photonics 2015, 9, 687694.

(15) Herz, L. M. How Lattice Dynamics Moderate the Electronic Properties of Metal-halide Perovskites. J. Phys. Chem. Lett. 2018, 9, 6853-6863.

(16) Saba, M.; Quochi, F.; Mura, A.; Bongiovanni, G. Excited State Properties of Hybrid Perovskites. Acc. Chem. Res. 2016, 49, 166-173.

(17) Miyata, K.; Atallah, T. L.; Zhu, X. Y. Lead Halide Perovskites: Crystal-liquid Duality, Phonon Glass Electron Crystals, and Large Polaron Formation. Sci. Adv. 2017, 3, e1701469.

(18) Bretschneider, S. A.; Ivanov, I.; Wang, H. I.; Miyata, K.; Zhu, X.; Bonn, M. Quantifying Polaron Formation and Charge Carrier Cooling in Lead-iodide Perovskites. Adv. Mater. 2018, 30, 1707312.

(19) Jha, A.; Duan, H.-G.; Tiwari, V.; Nayak, P. K.; Snaith, H. J.; Thorwart, M.; Miller, R. J. D. Direct Observation of Ultrafast Exciton Dissociation in Lead Iodide Perovskite by $2 \mathrm{D}$ Electronic Spectroscopy. ACS Photonics 2018, 5, 852-860.

(20) Bohn, B. J.; Simon, T.; Gramlich, M.; Richter, A. F.; Polavarapu, L.; Urban, A. S.; Feldmann, J. Dephasing and Quantum Beating of Excitons in Methylammonium Lead Iodide Perovskite Nanoplatelets. ACS Photonics 2018, 5, 648-654.

(21) Ghosh, T.; Aharon, S.; Etgar, L.; Ruhman, S. Free Carrier Emergence and Onset of Electron-phonon Coupling in Methylammonium Lead Halide Perovskite Films. J. Am. Chem. Soc. 2017, 139, 18262-18270.

(22) Nishida, J.; Breen, J. P.; Lindquist, K. P.; Umeyama, D.; Karunadasa, H. I.; Fayer, M. D. Dynamically Disordered Lattice in a Layered Pb-I-SCN Perovskite Thin Film Probed by Two-dimensional Infrared Spectroscopy. J. Am. Chem. Soc. 2018, 140, 9882-9890.

(23) Herz, L. M. Charge-carrier Dynamics in Organic-inorganic Metal Halide Perovskites. Annu. Rev. Phys. Chem. 2016, 67, 65-89.

(24) Oga, H.; Saeki, A.; Ogomi, Y.; Hayase, S.; Seki, S. Improved Understanding of the Electronic and Energetic Landscapes of Perovskite Solar Cells: High Local Charge Carrier Mobility, Eeduced Recombination, and Extremely Shallow Traps. J. Am. Chem. Soc. 2014, 136, 13818-13825.

(25) Wehrenfennig, C.; Liu, M.; Snaith, H. J.; Johnston, M. B.; Herz, L. M. Charge-carrier Dynamics in Vapour-deposited Films of the Organolead Halide Perovskite $\mathrm{CH}_{3} \mathrm{NH}_{3} \mathrm{PbI}_{3-\mathrm{x}} \mathrm{Cl}_{\mathrm{x}}$. Energy Environ. Sci. 2014, 7, 2269-2275.

(26) Zhu, H.; Miyata, K.; Fu, Y.; Wang, J.; Joshi, P. P.; Niesner, D.; Williams, K. W.; Jin, S.; Zhu, X. Y. Screening in Crystalline Liquids Protects Energetic Carriers in Hybrid Perovskites. Science 2016, 353, $1409-1413$

(27) Miyata, K.; Meggiolaro, D.; Trinh, M. T.; Joshi, P. P.; Mosconi, E.; Jones, S. C.; De Angelis, F.; Zhu, X.-Y. Large Polarons in Lead Halide Perovskites. Sci. Adv. 2017, 3, e1701217.

(28) Chen, T.; Chen, W.-L.; Foley, B. J.; Lee, J.; Ruff, J. P. C.; Ko, J. Y. P.; Brown, C. M.; Harriger, L. W.; Zhang, D.; Park, C.; Yoon, M.; Chang, Y.-M.; Choi, J. J.; Lee, S.-H. Origin of Long Lifetime of Bandedge Charge Carriers in Organic-inorganic Lead Iodide Perovskites. Proc. Natl. Acad. Sci. U. S. A. 2017, 114, 7519-7524.
(29) Batignani, G.; Fumero, G.; Srimath Kandada, A. R.; Cerullo, G.; Gandini, M.; Ferrante, C.; Petrozza, A.; Scopigno, T. Probing Femtosecond Lattice Displacement upon Photo-carrier Generation in Lead Halide Perovskite. Nat. Commun. 2018, 9, 1971.

(30) Wu, X.; Tan, L. Z.; Shen, X.; Hu, T.; Miyata, K.; Trinh, M. T.; Li, R.; Coffee, R.; Liu, S.; Egger, D. A.; Makasyuk, I.; Zheng, Q.; Fry, A.; Robinson, J. S.; Smith, M. D.; Guzelturk, B.; Karunadasa, H. I.; Wang, X.; Zhu, Z. Y.; Kronik, L.; Rappe, A. M.; Lindenberg, A. M. Light-induced Picosecond Rotational Disordering of the Inorganic Sublattice in Hybrid Perovskites. Sci. Adv. 2017, 3, e1602388.

(31) Ambrosio, F.; Wiktor, J.; De Angelis, F.; Pasquarello, A. Origin of Low Electron-hole Recombination Rate in Metal Halide Perovskites. Energy Environ. Sci. 2018, 11, 101-105.

(32) Zheng, F.; Tan, L. Z.; Liu, S.; Rappe, A. M. Rashba Spin-orbit Coupling Enhanced Carrier Lifetime in $\mathrm{CH}_{3} \mathrm{NH}_{3} \mathrm{PbI}_{3}$. Nano Lett. 2015, 15, 7794-7800.

(33) Zhai, Y.; Baniya, S.; Zhang, C.; Li, J.; Haney, P.; Sheng, C. X.; Ehrenfreund, E.; Vardeny, Z. V. Giant Rashba Splitting in 2D Organic-inorganic Halide Perovskites Measured by Transient Spectroscopies. Sci. Adv. 2017, 3, e1700704.

(34) Hutter, E. M.; Gelvez-Rueda, M. C.; Osherov, A.; Bulovic, V.; Grozema, F. C.; Stranks, S. D.; Savenije, T. J. Direct-indirect Character of the Bandgap in Methylammonium Lead Iodide Perovskite. Nat. Mater. 2017, 16, 115-120.

(35) Liu, S.; Zheng, F.; Koocher, N. Z.; Takenaka, H.; Wang, F.; Rappe, A. M. Ferroelectric Domain Wall Induced Band Gap Reduction and Charge Separation in Organometal Halide Perovskite. J. Phys. Chem. Lett. 2015, 6, 693-699.

(36) Bakulin, A. A.; Selig, O.; Bakker, H. J.; Rezus, Y. L. A.; Müller, C.; Glaser, T.; Lovrincic, R.; Sun, Z.; Chen, Z.; Walsh, A.; Frost, J. M.; Jansen, T. C. Real-time Observation of Organic Cation Reorientation in Methylammonium Lead Iodide Perovskites. J. Phys. Chem. Lett. 2015, 6, 3663-3669.

(37) Selig, O.; Sadhanala, A.; Müller, C.; Lovrincic, R.; Chen, Z.; Rezus, Y. L. A.; Frost, J. M.; Jansen, T. L. C.; Bakulin, A. A. Organic Cation Rotation and Immobilization in Pure and Mixed Methylammonium Lead-halide Perovskites. J. Am. Chem. Soc. 2017, 139, $4068-4074$

(38) Taylor, V. C. A.; Tiwari, D.; Duchi, M.; Donaldson, P. M.; Clark, I. P.; Fermin, D. J.; Oliver, T. A. A. Investigating the Role of the Organic Cation in Formamidinium Lead Iodide Perovskite using Ultrafast Spectroscopy. J. Phys. Chem. Lett. 2018, 9, 895-901.

(39) Park, M.; Kornienko, N.; Reyes-Lillo, S. E.; Lai, M.; Neaton, J. B.; Yang, P.; Mathies, R. A. Critical Role of Methylammonium Librational Motion in Methylammonium Lead Iodide $\left(\mathrm{CH}_{3} \mathrm{NH}_{3} \mathrm{PbI}_{3}\right)$ Perovskite Photochemistry. Nano Lett. 2017, 17, 4151-4157.

(40) Park, M.; Neukirch, A. J.; Reyes-Lillo, S. E.; Lai, M.; Ellis, S. R.; Dietze, D.; Neaton, J. B.; Yang, P.; Tretiak, S.; Mathies, R. A. Excitedstate Vibrational Dynamics toward the Polaron in Methylammonium Lead Iodide Perovskite. Nat. Commun. 2018, 9, 2525.

(41) Kim, H.; Hunger, J.; Canovas, E.; Karakus, M.; Mics, Z.; Grechko, M.; Turchinovich, D.; Parekh, S. H.; Bonn, M. Direct Observation of Mode-specific Phonon-band Gap Coupling in Methylammonium Lead Halide Perovskites. Nat. Commun. 2017, 8, 687.

(42) Richter, J. M.; Branchi, F.; Valduga de Almeida Camargo, F.; Zhao, B.; Friend, R. H.; Cerullo, G.; Deschler, F. Ultrafast Carrier Thermalization in Lead Iodide Perovskite Probed with Twodimensional Electronic Spectroscopy. Nat. Commun. 2017, 8, 376.

(43) Monahan, D. M.; Guo, L.; Lin, J.; Dou, L.; Yang, P.; Fleming, G. R. Room-temperature coherent optical phonon in $2 \mathrm{D}$ electronic spectra of $\left(\mathrm{CH}_{3} \mathrm{NH}_{3} \mathrm{PbI}_{3}\right)$ perovskite as a possible cooling bootleneck. J. Phys. Chem. Lett. 2017, 8, 3211-3215.

(44) Butkus, V.; Zigmantas, D.; Valkunas, L.; Abramavicius, D. Vibrational vs. Electronic Coherence in 2D Spectrum of Molecular Systems. Chem. Phys. Lett. 2012, 545, 40-43.

(45) Egorova, D. Self-analysis of Coherent Oscillations in Timeresolved Optical Signals. J. Phys. Chem. A 2014, 118, 10259-10267. 
(46) Guo, P.; Mannodi-Kanakkithodi, A.; Gong, J.; Xia, Y.; Stoumpos, C. C.; Cao, D. H.; Diroll, B. T.; Ketterson, J. B.; Wiederrecht, G. P.; Xu, T.; Chan, M. K. Y.; Kanatzidis, M. G.; Schaller, R. D. Infrared-pump Electronic-probe of Methylammonium Lead Iodide Reveals Electronically Decoupled Organic and Inorganic Sublattices. Nat. Commun. 2019, 10, 482.

(47) Cinquanta, E.; Meggiolaro, D.; Motti, S. G.; Gandini, M.; Alcocer, M. J. P.; Akkerman, Q. A.; Vozzi, C.; Manna, L.; De Angelis, F.; Petrozza, A.; Stagira, S. Ultrafast THz Probe of Photoinduced Polarons in Lead-halide Perovskite. Phys. Rev. Lett. 2019, 122, 166601.

(48) Neukirch, A. J.; Nie, W.; Blancon, J.; Appavoo, K.; Tsai, H.; Sfeir, M. Y.; Katan, C.; Pedesseau, L.; Even, J.; Crochet, J. J.; Gupta, G.; Mohite, A. D.; Tretiak, S. Polaron Stabilization by Cooperative Lattice Distortion and Cation Rotations in Hybrid Perovskite Materials. Nano Lett. 2016, 16, 3809-3816.

(49) Becke, A. D.; Edgecombe, K. E. A Simple Measure of Electron Localization in Atomic and Molecular Systems. J. Chem. Phys. 1990, 92, 5397.

(50) Stevens, W. J.; Basch, H.; Krauss, M. Compact Effective Potentials and Efficient Shared Exponent Basis Sets for the First and Second Row Atoms. J. Chem. Phys. 1984, 81, 6026.

(51) Praprotnik, M.; Janezic, D.; Mavri, J. Temperature Dependence of Water Vibrational Spectrum: a Molecular Dynamics Simulation Study. J. Phys. Chem. A 2004, 108, 11056-11062.

(52) Thomas, M.; Brehm, M.; Fligg, R.; Vöhringer, P.; Kirchner, B. Computing Vibrational Spectra from ab initio Molecular Dynamics. Phys. Chem. Chem. Phys. 2013, 15, 6608-6622.

(53) Mishra, P.; Vendrell, O.; Santra, R. Ultrafast Energy Transfer from Solvent to Solute Induced by Subpicosecond Highly Intense Thz Pulses. J. Phys. Chem. B 2015, 119, 8080-8086.

(54) Strachan, A. Normal Modes and Frequencies from Covariances in Molecular Dynamics or Monte Carlo Simulations. J. Chem. Phys. 2004, 120, 1-4.

(55) Madjet, M. E.-A.; Li, Z.; Vendrell, O. Ultrafast Hydrogen Migration in Acetylene Cation Driven by Non-adiabatic Effects. J. Chem. Phys. 2013, 138, 094311.

(56) Schmidt, W. M.; Baldridge, K. K.; Boatz, J. A.; Elbert, S. T.; Gordon, M. S.; Jensen, J. H.; Koseki, S.; Matsunaga, N.; Nguyen, N.; Su, S.; Windus, T. L.; Dupuis, M.; Montgomery, J. A., Jr General Atomic and Molecular Electronic Structure System. J. Comput. Chem. 1993, 14, 1347-1363.

(57) Ishikawa, T.; Hayes, S. A.; Keskin, S.; Corthey, G.; Hada, M.; Pichugin, K.; Marx, A.; Hirscht, J.; Shionuma, K.; Onda, K.; Okimoto, Y.; Koshihara, S.-y.; Yamamoto, T.; Cui, H.; Nomura, M.; Oshima, Y.; Abdel-Jawad, M.; Kato, R.; Miller, R. J. D. Direct Observation of Collective Modes Coupled to Molecular Orbital-driven Charge Transfer. Science 2015, 350, 1501.

(58) Prokhorenko, V. I.; Picchiotti, A.; Maneshi, S.; Miller, R. J. D. Broadband Electronic Two-dimensional Spectroscopy in the Deep UV. Springer Proc. Phys. 2015, 162, 432-435.

(59) Zhang, Y.; Huang, F.; Mi, Q. Preferential Facet Growth of Methylammonium Lead Halide Single Crystals Promoted by Halide Coordination. Chem. Lett. 2016, 45, 1030-1032.

(60) Noel, N. K.; Habisreutinger, S. N.; Wenger, B.; Klug, M. T.; Hörantner, M. T.; Johnston, M. B.; Nicholas, R. J.; Moore, D. T.; Snaith, H. J. A Low Viscosity, Low Boiling Point, Clean Solvent System for the Rapid Crystallisation of Highly Specular Perovskite Films. Energy Environ. Sci. 2017, 10, 145-152. 